Research Article

\title{
Commutators of Singular Integral Operators and Lipschitz Functions on Amalgam Spaces
}

\author{
Jiao Ma and Zongguang Liu (iD \\ China University of Mining and Technology, Beijing 100083, China \\ Correspondence should be addressed to Zongguang Liu; liuzg@cumtb.edu.cn \\ Received 16 August 2019; Revised 19 October 2019; Accepted 29 October 2019; Published 21 January 2020
}

Academic Editor: Dashan Fan

Copyright ( 2020 Jiao Ma and Zongguang Liu. This is an open access article distributed under the Creative Commons Attribution License, which permits unrestricted use, distribution, and reproduction in any medium, provided the original work is properly cited.

In this paper, we obtain the boundedness of commutators generated by singular integral operators and Lipschitz functions on amalgam spaces and weighted amalgam spaces.

\section{Introduction}

Let $b$ be a locally integrable function on $\mathbb{R}^{n}$ and $T_{\Omega}$ be a Calderón-Zygmund singular integral operator, then the commutator $\left[b, T_{\Omega}\right]$ generated by $b$ and $T_{\Omega}$ is defined as

$$
\left[b, T_{\Omega}\right] f(x)=b(x) T_{\Omega}(f)(x)-T_{\Omega}(b f)(x) .
$$

Coifman et al. [1] proved that $\left[b, T_{\Omega}\right]$ is bounded on $L^{p}\left(\mathbb{R}^{n}\right)$ for $1<p<\infty$ if and only if $b \in \mathrm{BMO}$. Then, Janson [2] obtained that $\left[b, T_{\Omega}\right]$ is bounded from $L^{p}$ to $L^{q}$ if and only if $b \in \operatorname{Lip}_{\beta}$, where $1<p<q<\infty, \quad 0<\beta<1$, and $(1 / q)=(1 / p)-(\beta / n)$. Recently, Wang [3] showed that $\left[b, T_{\Omega}\right]$ is bounded on weighted amalgam spaces when $b \in \mathrm{BMO}$. On the contrary, in [4], the boundedness for the commutator generated by the singular integral operator and the weighted Lipschitz function on Lebesgue spaces is obtained. The aim of this paper is to establish the boundedness of the commutator $\left[b, T_{\Omega}\right]$ generated by the Calderón-Zygmund singular integral operator and the weighted Lipschitz function on amalgam spaces.

Amalgam spaces were first introduced by Wiener $[5,6]$. He considered the special cases $\left(L^{1}, l^{2}\right),\left(L^{2}, l^{\infty}\right)$ and $\left(L^{\infty}, l^{1}\right),\left(L^{1}, l^{\infty}\right)$. A systematic study on general amalgam spaces $\left(L^{p}, l^{q}\right)$ goes back to the work of Holland [7]. He defined the spaces $\left(L^{p}, l^{q}\right)(1 \leq p, q \leq \infty)$ to be the sets of all locally $p$-th power summable functions $f$ on $\mathbb{R}$ such that

$$
\|f\|_{\left(L^{p}, l^{q}\right)}:=\left(\sum_{n=-\infty}^{\infty}\left(\int_{n}^{n+1}|f(x)|^{p} \mathrm{~d} x\right)^{q / p}\right)^{1 / q}<\infty
$$

with the usual convention applied when $p$ or $q$ is infinity.

Stewart [8] gave the definition of spaces $\left(L^{p}, l^{q}\right)$ over a locally compact Abelian group $G$. By the structure theorem, we may write $G=\mathbb{R}^{a} \times G_{1}$, where $a$ is a nonnegative integer and $G_{1}$ is a group which contains a compact open subgroup $H$ (if $G_{1}$ is compact, we take $H=G_{1}$; if $G_{1}$ is discrete and infinite, we take $H=\{0\}$; otherwise, $H$ is arbitrary but fixed). Let $I=[0,1)$ and $I_{\alpha}=\alpha+I$, where $\alpha=\left(n_{1}, \ldots, n_{a}, t\right)$, with $n_{l} \in \mathbb{Z}(l=1, \ldots, a)$ and $t \in G_{1}$ (the collection of $t$ 's being a transversal of $G_{1}$ in $H$, i.e., $G_{1}=\cup_{t}(t+H)$ is a coset decomposition of $\left.G_{1}\right)$. Then, in terms of the disjoint union $G=\cup_{\alpha \in J} I_{\alpha}$, where $J=\left\{\alpha: \alpha=\left(n_{1}, \ldots, n_{a}, t\right), n_{l} \in \mathbb{Z}\right.$ for $l=$ $1, \ldots, a$ and $\left.t \in G_{1}\right\}$, the amalgam space of $L^{p}$ and $l^{q}$ on $G$ was defined by

$$
\left(L^{p}, l^{q}\right)(G)=\left\{f:\|f\|_{p, q}<\infty\right\}
$$

where

$$
\begin{aligned}
\|f\|_{p, q} & =\sum_{\alpha \in J}\left(\|f\|_{L^{p}\left(I_{\alpha}\right)}^{q}\right)^{1 / q}, \quad 0<p \leq \infty, 0<q<\infty, \\
\|f\|_{p, \infty} & =\sup _{\alpha \in J}\|f\|_{L^{p}\left(I_{\alpha}\right)}, \quad 0<p \leq \infty .
\end{aligned}
$$


We refer the reader to the work of Fournier and Steward [9] for more information about these spaces.

Fofana introduced in [10] the amalgam spaces $\left(L^{p}, L^{q}\right)^{\alpha}\left(\mathbb{R}^{n}\right)$ as follows:
$\left(L^{p}, L^{q}\right)^{\alpha}\left(\mathbb{R}^{n}\right)=\left\{f \in L_{\mathrm{loc}}^{p}\left(\mathbb{R}^{n}\right):\|f\|_{\left(L^{p}, L^{q}\right)^{\alpha}\left(\mathbb{R}^{n}\right)}<\infty\right\}, \quad 1 \leq p, q, \alpha \leq \infty$,

where

$$
\|f\|_{\left(L^{p}, L^{q}\right)^{\alpha}\left(\mathbb{R}^{n}\right)}=\sup _{r>0}\left\||B(y, r)|^{(1 / \alpha)-(1 / p)-(1 / q)}\right\| f \chi_{B(y, r)}\left\|L^{p}\left(\mathbb{R}^{n}\right)\right\|_{L^{q}\left(\mathbb{R}^{n}\right)^{\prime}}
$$

here $B(y, r)=\left\{x \in \mathbb{R}^{n}:|x-y|<r\right\}$ and $|B(y, r)|$ is its Lebesgue measure. In [10], the author showed that the space $\left(L^{p}, L^{q}\right)^{\alpha}\left(\mathbb{R}^{n}\right)$ is nontrivial if and only if $p \leq \alpha \leq q$; thus, we will always assume $p \leq \alpha \leq q$ throughout the present article.

We recall the following interesting facts about the amalgam spaces $\left(L^{p}, L^{q}\right)^{\alpha}\left(\mathbb{R}^{n}\right)$ :

(1) For $1 \leq p \leq \alpha \leq q \leq \infty$, we can easily see that $\left(L^{p}, L^{q}\right)^{\alpha}\left(\mathbb{R}^{n}\right) \subseteq\left(L^{p}, L^{q}\right)\left(\mathbb{R}^{n}\right)$, where $\left(L^{p}, L^{q}\right)\left(\mathbb{R}^{n}\right)$ is Wiener's amalgam space (see [7])

(2) If $1 \leq p<\alpha$ and $q=\infty$, then $\left(L^{p}, L^{q}\right)^{\alpha}\left(\mathbb{R}^{n}\right)$ is just the classical Morrey space $L^{p, 1-(p / \alpha)}\left(\mathbb{R}^{n}\right)$

(3) If $p=\alpha$ and $q=\infty$, then $\left(L^{p}, L^{q}\right)^{\alpha}\left(\mathbb{R}^{n}\right)$ is just the usual Lebesgue space $L^{p}\left(\mathbb{R}^{n}\right)$

The first main result of this paper is as follows.

Theorem 1. Let $1<p<q<\infty, 0<\gamma<1, \quad(1 / q)=(1 / p)-$ $(\gamma / n)$, and $1 / \beta=(1 / \alpha)-(\gamma / n)$. Assume that $p<\alpha<s<\infty$ and $q<\beta<s<\infty$. If $b \in$ Lip $_{\gamma}$, then $\left[b, T_{\Omega}\right]$ is bounded from $\left(L^{p}, L^{s}\right)^{\alpha}\left(\mathbb{R}^{n}\right)$ to $\left(L^{q}, L^{s}\right)^{\beta}\left(\mathbb{R}^{n}\right)$.

We also need the definition of the weighted amalgam spaces.

Definition 1 (see [3]). Let $1 \leq p \leq \alpha \leq q \leq \infty$ and $w$ and $\mu$ be two weights on $\mathbb{R}^{n}$. Then, the weighted amalgam spaces $\left(L^{p}, L^{q}\right)^{\alpha}(w ; \mu)$ are defined as

$$
\left(L^{p}, L^{q}\right)^{\alpha}(w ; \mu)=\left\{f \in L_{\mathrm{loc}}^{p}(w):\|f\|_{\left(L^{p}, L^{q}\right)^{\alpha}(w ; \mu)}<\infty\right\},
$$

where

$$
\|f\|_{\left(L^{p}, L^{q}\right)^{\alpha}(w ; \mu)}=\sup _{r>0}\left\|w(B(y, r))^{(1 / \alpha)-(1 / p)-(1 / q)}\right\| f \chi_{B(y, r)}\left\|_{L_{w}^{p}}\right\|_{L_{\mu}^{q}},
$$

with the usual modification when $q=\infty$. Moreover, the spaces defined above are Banach spaces.

Definition 2 (see [3]). Let $1 \leq p \leq \alpha \leq q \leq \infty$ and $u$, $v$, and $\mu$ be three weights on $\mathbb{R}^{n}$. Then, the weighted amalgam spaces $\left(L^{p}, L^{q}\right)^{\alpha}(\nu, u ; \mu)$ are defined as

$$
\left(L^{p}, L^{q}\right)^{\alpha}(v, u ; \mu)=\left\{f \in L_{\mathrm{loc}}^{p}(v):\|f\|_{\left(L^{p}, L^{q}\right)^{\alpha}(v, u ; \mu)}<\infty\right\},
$$

where

$$
\|f\|_{\left(L^{p}, L^{q}\right)^{\alpha}(v, \mathcal{u} ; \mu)}=\sup _{r>0}\left\|\mathcal{u}(B(y, r))^{(1 / \alpha)-(1 / p)-(1 / q)}\right\| f \chi_{B(y, r)}\left\|_{L_{v}^{p}}\right\|_{L_{\mu}^{q}},
$$

with the usual modification when $q=\infty$. Moreover, the spaces defined above are Banach spaces.

The second main result is the following:

Theorem 2. Let $1<p<q<\infty, \quad 0<\beta<1, \quad p<\alpha_{1}<s<\infty$, $q<\alpha_{2}<s<\infty,(1 / q)=(1 / p)-(\beta / n)$, and $\left(1 / \alpha_{2}\right)=\left(1 / \alpha_{1}\right)-$ $(\beta / n)$. Suppose that $u \in A_{1}, v$ is a weight, and $b \in \operatorname{Lip}_{\beta, u}$, then $\left[b, T_{\Omega}\right]$ is bounded from $\left(L^{p}, L^{s}\right)^{\alpha_{1}}\left(u, u^{1-q} ; \nu\right)$ to $\left(L^{q}, L^{s}\right)^{\alpha_{2}}\left(u^{1-q} ; \nu\right)$.

Throughout the paper, we will assume that the Calderón-Zygmund singular integral operator $T_{\Omega}$ is defined by

$$
T_{\Omega} f(x)=p \cdot v \cdot \int_{\mathbb{R}^{n}} \frac{\Omega(x-y)}{|x-y|^{n}} f(y) \mathrm{d} y,
$$

where $\Omega$ is a homogeneous function of degree zero, $\int_{\delta^{n-1}} \Omega\left(x^{\prime}\right) \mathrm{d} \sigma\left(x^{\prime}\right)=0$ and $\Omega \in C^{\infty}\left(\delta^{n-1}\right)$.

1.1. Some Preliminaries and Notations. We denote by $\Delta_{h}^{k}$ the $k^{\text {th }}$ difference operator. That is,

$$
\begin{aligned}
\Delta_{h}^{1} f(x) & =\Delta_{h} f(x)=f(x+h)-f(x), \\
\Delta_{h}^{k+1} f(x) & =\Delta_{h}^{k} f(x+h)-\Delta_{h}^{k} f(x), \quad k \geq 1 .
\end{aligned}
$$

For a cube $Q$ and a locally integrable function $f$, we write

$$
f_{Q}=\frac{1}{|Q|} \int_{Q} f(x) \mathrm{d} x \text {. }
$$

For $\beta>0$, the Lipschitz space $\Lambda_{\beta}$ consists of functions $f$ such that

$$
\|f\|_{\Lambda_{\beta}}=\sup _{x, h \in \mathbb{R}^{n}, h \neq 0} \frac{\left|\Delta_{h}^{[\beta]+1} f(x)\right|}{|h|^{\beta}}<\infty .
$$

For $\beta>0$ and $f \in L_{\text {loc }}^{1}$, we say $f \in \operatorname{Lip}_{\beta}$ if there is a constant $C>0$ such that

$$
|f(x)-f(y)| \leq C|x-y|^{\beta},
$$

for any $x, y \in \mathbb{R}^{n}$. The minimal constant $C$ mentioned above is called the $\operatorname{Lip}_{\beta}$ norm of $f$, denoted by $\|f\|_{\operatorname{Lip}_{\beta}}$.

Remark 1. When $0<\beta<1, \quad \Lambda_{\beta}=\operatorname{Lip}_{\beta}$; when $\beta \geq 1$, $\operatorname{Lip}_{\beta} \subsetneq \Lambda_{\beta}$. 
Lemma 1 (see [11]). If $0<\beta<1$ and $1 \leq p<\infty$, then

$$
\begin{aligned}
\|f\|_{\operatorname{Lip}_{\beta}} & =\sup _{Q} \frac{1}{|Q|^{1+(\beta / n)}} \int_{Q}\left|f(x)-f_{Q}\right| \mathrm{d} x \\
& \approx \sup _{Q} \frac{1}{|Q|^{(\beta / n)}}\left(\frac{1}{|Q|} \int_{Q}\left|f(x)-f_{Q}\right|^{p} \mathrm{~d} x\right)^{1 / p} .
\end{aligned}
$$

Lemma 2 (see [11]). If $Q^{*} \subseteq Q$, then $\left|f_{Q^{*}}-f_{Q}\right|$ $\leq C\|f\|_{L i p_{\beta}}|Q|^{\beta / n}$.

From Lemma 1, we see that

$$
\left|f(y)-f_{\mathrm{Q}}\right| \leq C\|f\|_{\operatorname{Lip}_{\beta}}|Q|^{\beta / n}, \quad \text { a.e. } y \in Q \text {. }
$$

The following result was established in [2].

Theorem 3 (see [2]). Let $0<\beta<1$ and $1 \leq p<\infty$. Then, the following statements are equivalent:

(a) $b \in \operatorname{Lip}_{\beta}$

(b) $\left[b, T_{\Omega}\right]$ is bounded from $L^{p}\left(\mathbb{R}^{n}\right)$ to $L^{q}\left(\mathbb{R}^{n}\right)$, where $(1 / q)=(1 / p)-(\beta / n)$

$A$ weight $w$ is said to belong to the Muckenhoupt class $A_{p}$ for $1<p<\infty,(1 / p)+\left(1 / p^{\prime}\right)=1$, if there exists a constant $C$ such that

$$
\left(\frac{1}{|B|} \int_{B} w(x) \mathrm{d} x\right)\left(\frac{1}{|B|} \int_{B} w(x)^{1-p^{\prime}} \mathrm{d} x\right)^{p-1} \leq C,
$$

for any ball $B \subset \mathbb{R}^{n}$. The class $A_{1}$ is defined by replacing the above inequality with

$$
\frac{1}{|B|} \int_{B} w(x) \mathrm{d} x \leq C w(x), \quad \text { a.e. } x \in B,
$$

for any ball $B \subset \mathbb{R}^{n}$ and $A_{\infty}=\cup_{p \geq 1} A_{p}$ (see [12]).

Let $0<\beta<1$ and $w$ be a weight function, then the weighted Lipschitz space $\operatorname{Lip}_{\beta, w}$ is defined by

$$
\operatorname{Lip}_{\beta, w}=\left\{f \in L_{\mathrm{loc}}^{1}\left(\mathbb{R}^{n}\right):\|f\|_{\operatorname{Lip}_{\beta, w}}<\infty\right\},
$$

where

$$
\|f\|_{\operatorname{Lip}_{\beta, w}}=\sup _{B} \frac{1}{w(B)^{1+(\beta / n)}} \int_{B}\left|f(y)-f_{B}\right| \mathrm{d} y .
$$

Lemma 3 (see $[4,12]$ ). If $0<\beta<1, u \in A_{1}, b \in \operatorname{Lip}_{\beta, u}$, and $1 \leq p \leq \infty$, then we have

$$
\|b\|_{\operatorname{Lip}_{\beta, u}} \approx \sup _{Q} u(Q)^{-(\beta / n)}\left(u(Q)^{-1} \int_{Q}\left|b(x)-b_{B}\right|^{p} u(x)^{1-p} \mathrm{~d} x\right)^{1 / p} .
$$

Lemma 4 (see $[4,12]$ ). If $0<\beta<1, u \in A_{1}, b \in \operatorname{Lip}_{\beta, u}$, and $Q$ is a cube, then there exists a constant $C>0$, independent of $b$ and $Q$, such that

$$
\sup _{x \in Q}\left|b(x)-b_{Q}\right| \leq C\|b\|_{\operatorname{Lip}_{\beta, u}} u(Q)^{1+(\beta / n)}|Q|^{-1} .
$$

Lemma 5 (see $[4,12]$ ). If $0<\beta<1, u \in A_{1}, b \in \operatorname{Lip}_{\beta, u}$, and $Q$ is a cube, then there exists a constant $C>0$, independent of $b$ and $Q$, such that

$$
\left|b_{2^{k} Q}-b_{Q}\right| \leq C k u(x) u\left(2^{k} Q\right)^{\beta / n}\|b\|_{\operatorname{Lip}_{\beta, u}}, \quad \text { for all } x \in Q \text {. }
$$

Theorem 4 (see [4]). Let $u \in A_{1},(1 / q)=(1 / p)-(\beta / n)$ for $0<\beta<1$ and $1<p<q<\infty$. Then, $b \in \operatorname{Lip}_{\beta, u}$ if and only if $\left[b, T_{\Omega}\right]$ is bounded from $L^{p}(u)$ to $L^{q}\left(u^{1-q}\right)$.

\subsection{Proofs of the Main Results}

Proof of Theorem 1. Let $1<p<q<\infty, 0<\gamma<1, p<\alpha<s$, $q<\beta<s,(1 / q)=(1 / p)-(\gamma / n),(1 / \beta)=(1 / \alpha)-(\gamma / n)$, and $f \in\left(L^{p}, L^{s}\right)^{\alpha}\left(\mathbb{R}^{n}\right)$. Fix $y \in \mathbb{R}^{n}$ and $r>0$ and set $B=B(y, r)$ and $2 B=B(y, 2 r)$. Let $f_{1}=f \chi_{2 B}$ and $f_{2}=f-f_{1}$. We write

$$
\begin{aligned}
& |B(y, r)|^{(1 / \beta)-(1 / q)-(1 / s)}\left\|\left[b, T_{\Omega}\right] f \chi_{B(y, r)}\right\|_{L^{q}} \\
& \leq|B(y, r)|^{(1 / \beta)-(1 / q)-(1 / s)}\left\|\left[b, T_{\Omega}\right] f_{1} \chi_{B(y, r)}\right\|_{L^{q}} \\
& \quad+|B(y, r)|^{(1 / \beta)-(1 / q)-(1 / s)}\left\|\left[b, T_{\Omega}\right] f_{2} \chi_{B(y, r)}\right\|_{L^{q}} \\
& =: J_{1}(y, r)+J_{2}(y, r) .
\end{aligned}
$$

We will estimate $J_{1}(y, r)$ and $J_{2}(y, r)$ separately. Using Theorem 3, we have

$$
\begin{aligned}
J_{1}(y, r) & \leq|B(y, r)|^{1 / \beta-(1 / q)-(1 / s)}\left\|\left[b, T_{\Omega}\right] f_{1}\right\|_{L^{q}} \\
& \leq C|B(y, r)|^{(1 / \beta)-(1 / q)-(1 / s)}\left\|f \chi_{2 B}\right\|_{L^{p}} \\
& =C|B(y, 2 r)|^{(1 / \alpha)-(1 / p)-(1 / s)}\left\|f \chi_{2 B}\right\|_{L^{p}} \frac{|B(y, r)|^{(1 / \beta)-(1 / q)-(1 / s)}}{|B(y, 2 r)|^{(1 / \alpha)-(1 / p)-(1 / s)}} .
\end{aligned}
$$

Since $(1 / q)=(1 / p)-(\gamma / n)$ and $(1 / \beta)=(1 / \alpha)-(\gamma / n)$, we have $(1 / \alpha)-(1 / p)-(1 / s)=(1 / \beta)-(1 / q)-(1 / s)<0$. Consequently,

$$
J_{1}(y, r) \leq C|B(y, 2 r)|^{(1 / \alpha)-(1 / p)-(1 / s)}\left\|f \chi_{2 B}\right\|_{L^{p}} .
$$

Now we treat $J_{2}(y, r)$. It is obvious that $|x-z| \approx|y-z|$, when $x \in B(y, r)$ and $z \in B(y, 2 r)^{c}$. We have

$$
\begin{aligned}
\left|\left[b, T_{\Omega}\right] f_{2}(x)\right| \leq & \left|\left(b(x)-b_{B}\right) T_{\Omega} f_{2}(x)\right| \\
& +\left|T_{\Omega}\left(\left(b-b_{B}\right) f_{2}\right)(x)\right| .
\end{aligned}
$$

Decomposing $\mathbb{R}^{n}$ into a geometrically increasing sequence of concentric balls, we have 


$$
\begin{aligned}
& \left|T_{\Omega} f_{2}(x)\right| \leq C \int_{\mathbb{R}^{n}} \frac{\left|f_{2}(z)\right|}{|x-z|^{n}} \mathrm{~d} z \leq C \int_{B(y, 2 r)^{c}} \frac{|f(z)|}{|y-z|^{n}} \mathrm{~d} z \\
& =C \sum_{j=1}^{\infty} \int_{B\left(y, 2^{j+1} r\right) \backslash B\left(y, 2^{j} r\right)} \frac{|f(z)|}{|y-z|^{n}} \mathrm{~d} z \\
& \leq C \sum_{j=1}^{\infty} \frac{1}{\left|B\left(y, 2^{j+1} r\right)\right|} \int_{B\left(y, 2^{j+1} r\right)}|f(z)| \mathrm{d} z \\
& \left|T_{\Omega}\left(\left(b-b_{B}\right) f_{2}\right)(x)\right|=\left|\int_{\mathbb{R}^{n}}\left(b(z)-b_{B}\right) f_{2}(z) \frac{\Omega(x-z)}{|x-z|^{n}} \mathrm{~d} z\right| \\
& \leq C \int_{(2 B)^{c}}\left|b(z)-b_{B}\right||f(z)| \frac{1}{|x-z|^{n}} \mathrm{~d} z \\
& \leq C \sum_{j=1}^{\infty} \frac{1}{\left|B\left(y, 2^{j+1} r\right)\right|} \int_{B\left(y, 2^{j+1} r\right)}\left|b(z)-b_{B}\right||f(z)| \mathrm{d} z .
\end{aligned}
$$

From these estimates, it follows that

$$
\begin{aligned}
J_{2}(y, r)= & |B(y, r)|^{(1 / \beta)-(1 / q)-(1 / s)}\left(\int_{B(y, r)}\left|\left[b, T_{\Omega}\right] f_{2}(x)\right|^{q} \mathrm{~d} x\right)^{1 / q} \\
\leq & C|B(y, r)|^{(1 / \beta)-(1 / q)-(1 / s)} \sum_{j=1}^{\infty} \frac{1}{\left|B\left(y, 2^{j+1} r\right)\right|} \int_{B\left(y, 2^{j+1} r\right)}|f(z)| \mathrm{d} z \times\left(\int_{B(y, r)}\left|b(x)-b_{B}\right|^{q} d x\right)^{1 / q} \\
& +C|B(y, r)|^{(1 / \beta)-(1 / s)} \sum_{j=1}^{\infty} \frac{1}{\left|B\left(y, 2^{j+1} r\right)\right|} \int_{B\left(y, 2^{j+1} r\right)}\left|b(z)-b_{B}\right||f(z)| \mathrm{d} z \\
= & J_{3}(y, r)+J_{4}(y, r) .
\end{aligned}
$$

Using Hölder's inequality and Lemma 1, we have

$$
\begin{aligned}
J_{3}(y, r) & \leq C\|b\|_{\operatorname{Lip}_{\gamma}}|B(y, r)|^{(1 / \beta)-(1 / q)-(1 / s)} \sum_{j=1}^{\infty} \frac{1}{\left|B\left(y, 2^{j+1} r\right)\right|} \int_{B\left(y, 2^{j+1} r\right)}|f(z)| \mathrm{d} z|B(y, r)|^{1 / p} \\
& \leq C\|b\|_{\operatorname{Lip}_{\gamma}} \sum_{j=1}^{\infty}|B(y, r)|^{(1 / \beta)-(1 / q)-(1 / s)+(1 / p)}\left(\frac{1}{\left|B\left(y, 2^{j+1} r\right)\right|} \int_{B\left(y, 2^{j+1} r\right)}|f(z)|^{p} \mathrm{~d} z\right)^{1 / p} \\
& =C\|b\|_{\operatorname{Lip}_{\gamma}} \sum_{j=1}^{\infty}\left|B\left(y, 2^{j+1} r\right)\right|^{(1 / \alpha)-(1 / p)-(1 / s)}\left\|f \chi_{B\left(y, 2^{j+1} r\right)}\right\|_{L^{p}} \frac{|B(y, r)|^{(1 / \alpha)-(1 / s)}}{\left|B\left(y, 2^{j+1} r\right)\right|^{(1 / \alpha)-(1 / s)}} \\
& =C\|b\|_{\operatorname{Lip}_{\gamma}} \sum_{j=1}^{\infty}\left|B\left(y, 2^{j+1} r\right)\right|^{(1 / \alpha)-(1 / p)-(1 / s)}\left\|f \chi_{B\left(y, 2^{j+1} r\right)}\right\|_{L^{p}}\left(\frac{1}{2^{(j+1) n}}\right)^{(1 / \alpha)-(1 / s)} \cdot
\end{aligned}
$$


As for $J_{4}(y, r)$, we get

$$
\begin{aligned}
J_{4}(y, r) \leq & C|B(y, r)|^{(1 / \beta)-(1 / s)} \sum_{j=1}^{\infty} \frac{1}{\left|B\left(y, 2^{j+1} r\right)\right|} \times \int_{B\left(y, 2^{j+1} r\right)}\left(\left|b(z)-b_{2^{j+1} B}\right|+\left|b_{2^{j+1} B}-b_{B}\right|\right)|f(z)| \mathrm{d} z \\
= & C|B(y, r)|^{(1 / \beta)-(1 / s)} \sum_{j=1}^{\infty} \frac{1}{\left|B\left(y, 2^{j+1} r\right)\right|} \int_{B\left(y, 2^{j+1} r\right)}\left|b(z)-b_{2^{j+1} B}\right||f(z)| \mathrm{d} z \\
& +C|B(y, r)|^{(1 / \beta)-(1 / s)} \sum_{j=1}^{\infty} \frac{1}{\left|B\left(y, 2^{j+1} r\right)\right|} \int_{B\left(y, 2^{j+1} r\right)}\left|b_{2^{j+1} B}-b_{B}\right||f(z)| \mathrm{d} z \\
= & J_{5}(y, r)+J_{6}(y, r) .
\end{aligned}
$$

It follows from Hölder's inequality and Lemma 1 that

$$
\begin{aligned}
J_{5}(y, r) & \leq C|B(y, r)|^{(1 / \beta)-(1 / s)} \sum_{j=1}^{\infty} \frac{1}{\left|B\left(y, 2^{j+1} r\right)\right|}\left(\int_{B\left(y, 2^{j+1} r\right)}|f(z)|^{p} \mathrm{~d} z\right)^{1 / p} \times\left(\int_{B\left(y, 2^{j+1} r\right)}\left|b(z)-b_{2^{j+1} B}\right|^{p^{\prime}} \mathrm{d} z\right)^{1 / p^{\prime}} \\
& \leq C\|b\|_{\operatorname{Lip}_{\gamma}}|B(y, r)|^{(1 / \beta)-(1 / s)} \sum_{j=1}^{\infty}\left\|f \chi_{B\left(y, 2^{j+1} r\right)}\right\|_{L^{p}}\left|B\left(y, 2^{j+1} r\right)\right|^{(y / n)-(1 / p)} \\
& =C\|b\|_{\operatorname{Lip}_{\gamma}} \sum_{j=1}^{\infty}\left|B\left(y, 2^{j+1} r\right)\right|^{(1 / \alpha)-(1 / p)-(1 / s)}\left\|f \chi_{B\left(y, 2^{j+1} r\right)}\right\|_{L^{p}} \frac{|B(y, r)|^{(1 / \beta)-(1 / s)}}{\left|B\left(y, 2^{j+1} r\right)\right|^{(1 / \beta)-(1 / s)}} \\
& =C\|b\|_{\operatorname{Lip}_{\gamma}} \sum_{j=1}^{\infty}\left|B\left(y, 2^{j+1} r\right)\right|^{(1 / \alpha)-(1 / p)-(1 / s)}\left\|f \chi_{B\left(y, 2^{j+1} r\right)}\right\|_{L^{p}}\left(\frac{1}{2^{(j+1) n}}\right)^{(1 / \beta)-(1 / s)} \cdot
\end{aligned}
$$

For the last term $J_{6}(y, r)$, we use Hölder's inequality and Lemma 2 to get

$$
\begin{aligned}
J_{6}(y, r) & \leq C|B(y, r)|^{(1 / \beta)-(1 / s)} \sum_{j=1}^{\infty}\|b\|_{\operatorname{Lip}_{\gamma}}\left|B\left(y, 2^{j+1} r\right)\right|^{\gamma / n} \frac{1}{\left|B\left(y, 2^{j+1} r\right)\right|} \int_{B\left(y, 2^{j+1} r\right)}|f(z)| \mathrm{d} z \\
& \leq C\|b\|_{\operatorname{Lip}_{\gamma}} \sum_{j=1}^{\infty}|B(y, r)|^{(1 / \beta)-(1 / s)}\left(\int_{B\left(y, 2^{j+1} r\right)}|f(z)|^{p} \mathrm{~d} z\right)^{1 / p} \cdot\left|B\left(y, 2^{j+1} r\right)\right|^{(\gamma / n)-(1 / p)} \\
& =C\|b\|_{\operatorname{Lip}_{\gamma}} \sum_{j=1}^{\infty}\left|B\left(y, 2^{j+1} r\right)\right|^{(1 / \alpha)-(1 / p)-(1 / s)}\left\|f \chi_{B\left(y, 2^{j+1} r\right)}\right\|_{L^{p}} \frac{|B(y, r)|^{(1 / \beta)-(1 / s)}}{\left|B\left(y, 2^{j+1} r\right)\right|^{(1 / \beta)-(1 / s)}} \\
& =C\|b\|_{\operatorname{Lip}_{\gamma}} \sum_{j=1}^{\infty}\left|B\left(y, 2^{j+1} r\right)\right|^{(1 / \alpha)-(1 / p)-(1 / s)}\left\|f \chi_{B\left(y, 2^{j+1} r\right)}\right\|_{L^{p}}\left(\frac{1}{2^{(j+1) n}}\right)^{(1 / \beta)-(1 / s)} \cdot
\end{aligned}
$$


Summarizing the above estimates, we conclude that

$$
\begin{aligned}
J_{2}(y, r) \leq & C \sum_{j=1}^{\infty}\left|B\left(y, 2^{j+1} r\right)\right|^{(1 / \alpha)-(1 / p)-(1 / s)}\left\|f \chi_{B\left(y, 2^{j+1} r\right)}\right\|_{L^{p}}\left(\frac{1}{2^{(j+1) n}}\right)^{(1 / \alpha)-(1 / s)} \\
& +C \sum_{j=1}^{\infty}\left|B\left(y, 2^{j+1} r\right)\right|^{(1 / \alpha)-(1 / p)-(1 / s)}\left\|f \chi_{B\left(y, 2^{j+1} r\right)}\right\|_{L^{p}}\left(\frac{1}{2^{(j+1) n}}\right)^{(1 / \beta)-(1 / s)} .
\end{aligned}
$$

Taking the $L^{s}$-norm and using Minkowski's inequality, we get

$$
\begin{aligned}
& \left\||B(y, r)|^{(1 / \beta)-(1 / q)-(1 / s)}\right\|\left[b, T_{\Omega}\right] f \chi_{B(y, r)}\left\|_{L^{q}}\right\|_{L^{s}} \\
& \leq\left\|J_{1}\right\|_{L^{s}}+\left\|J_{2}\right\|_{L^{s}} \\
& \leq C\left\||B(y, 2 r)|^{(1 / \alpha)-(1 / p)-(1 / s)}\right\| f \chi_{2 B}\left\|_{L^{p}}\right\|_{L^{s}} \\
& \quad+C \sum_{j=1}^{\infty}\left\|\left|B\left(y, 2^{j+1} r\right)\right|^{(1 / \alpha)-(1 / p)-(1 / s)}\right\| f \chi_{B}\left(y, 2^{j+1} r\right)\left\|_{L^{p}}\right\|_{L^{s}}\left(\frac{1}{2^{(j+1) n}}\right)^{(1 / \alpha)-(1 / s)} \\
& \quad+C \sum_{j=1}^{\infty}\left\|\left|B\left(y, 2^{j+1} r\right)\right|^{(1 / \alpha)-(1 / p)-(1 / s)}\right\| f \chi_{B\left(y, 2^{j+1} r\right)}\left\|_{L^{p}}\right\| L_{L^{s}}\left(\frac{1}{2^{(j+1) n}}\right)^{(1 / \beta)-(1 / s)} \\
& \leq C\|f\|_{\left(L^{p}, L^{s}\right)^{\alpha}\left(\mathbb{R}^{n}\right) \cdot}
\end{aligned}
$$

Thus, by taking the supremum over all $r>0$, the proof is completed.

Proof of Theorem 2. Let $1<p<q<\infty, 0<\beta<1, p<\alpha_{1}<s$, $q<\alpha_{2}<s, \quad(1 / q)=(1 / p)-(\beta / n), \quad\left(1 / \alpha_{2}\right)=\left(1 / \alpha_{1}\right)-(\beta / n)$, and $f \in\left(L^{p}, L^{s}\right)^{\alpha_{1}}\left(u, u^{1-q} ; \nu\right)$. Fix $y \in \mathbb{R}^{n}$ and $r>0$ and set $B=B(y, r)$ and $2 B=B(y, 2 r)$. Let $f_{1}=f \chi_{2 B}$ and $f_{2}=f-f_{1}$. We write

$$
\begin{aligned}
& u^{1-q}(B(y, r))^{\left(1 / \alpha_{2}\right)-(1 / q)-(1 / s)}\left\|\left[b, T_{\Omega}\right] f \chi_{B(y, r)}\right\|_{L^{q}\left(u^{1-q}\right)} \\
& \leq u^{1-q}(B(y, r))^{\left(1 / \alpha_{2}\right)-(1 / q)-(1 / s)}\left\|\left[b, T_{\Omega}\right] f_{1} \chi_{B(y, r)}\right\|_{L^{q}\left(u^{1-q}\right)} \\
& \quad+u^{1-q}(B(y, r))^{\left(1 / \alpha_{2}\right)-(1 / q)-(1 / s)}\left\|\left[b, T_{\Omega}\right] f_{2} \chi_{B(y, r)}\right\|_{L^{q}\left(u^{1-q}\right)} \\
& =: K_{1}(y, r)+K_{2}(y, r) .
\end{aligned}
$$

Now we will estimate $K_{1}(y, r)$ and $K_{2}(y, r)$ separately. Using Theorem 4, we have

$$
\begin{aligned}
& K_{1}(y, r) \leq u^{1-q}(B(y, r))^{\left(1 / \alpha_{2}\right)-(1 / q)-(1 / s)}\left\|\left[b, T_{\Omega}\right] f_{1}\right\|_{L^{q}\left(u^{1-q}\right)} \\
& \leq C u^{1-q}(B(y, r))^{\left(1 / \alpha_{2}\right)-(1 / q)-(1 / s)}\left\|f \chi_{2 B}\right\|_{L^{p}(u)} \\
& =C u^{1-q}(B(y, 2 r))^{\left(1 / \alpha_{1}\right)-(1 / p)-(1 / s)}\left\|f \chi_{2 B}\right\|_{L^{p}(u)} \frac{u^{1-q}(B(y, r))^{\left(1 / \alpha_{2}\right)-(1 / q)-(1 / s)}}{u^{1-q(B(y, 2 r))^{\left(1 / \alpha_{1}\right)-(1 / p)-(1 / s)}} .}
\end{aligned}
$$


Noticing that $u^{1-q} \in A_{\infty}$ implies that $u^{1-q}$ is a doubling measure and that $(1 / q)=(1 / p)-(\beta / n)$ and $\left(1 / \alpha_{2}\right)=\left(1 / \alpha_{1}\right)-(\beta / n)$, and it follows that

$$
\frac{u^{1-q}(B(y, r))^{\left(1 / \alpha_{2}\right)-(1 / q)-(1 / s)}}{u^{1-q}(B(y, 2 r))^{\left(1 / \alpha_{1}\right)-(1 / q)-(1 / s)}} \leq C .
$$

$$
K_{1}(y, r) \leq C u^{1-q}(B(y, 2 r))^{\left(1 / \alpha_{1}\right)-(1 / p)-(1 / s)}\left\|f \chi_{2 B}\right\|_{L^{p}(u)} .
$$

Similar to the proof of Theorem 1, we decompose $K_{2}(y, r)$ as

So we have

$$
\begin{aligned}
K_{2}(y, r)= & u^{1-q}(B(y, r))^{\left(1 / \alpha_{2}\right)-(1 / q)-(1 / s)}\left\|\left[b, T_{\Omega}\right] f_{2} \chi_{B(y, r)}\right\|_{L^{q}\left(u^{1-q}\right)} \\
\leq & C u^{1-q}(B(y, r))^{\left(1 / \alpha_{2}\right)-(1 / q)-(1 / s)} \sum_{j=1}^{\infty} \frac{1}{\left|B\left(y, 2^{j+1} r\right)\right|} \int_{B\left(y, 2^{j+1} r\right)}|f(z)| \mathrm{d} z \\
& \times\left(\int_{B(y, r)}\left|b(x)-b_{B}\right|^{q} u^{1-q}(x) \mathrm{d} x\right)^{1 / q}+C u^{1-q}(B(y, r))^{\left(1 / \alpha_{2}\right)-(1 / s)} \sum_{j=1}^{\infty} \frac{1}{\left|B\left(y, 2^{j+1} r\right)\right|} \int_{B\left(y, 2^{j+1} r\right)}\left|b(z)-b_{B}\right||f(z)| \mathrm{d} z \\
= & K_{3}(y, r)+K_{4}(y, r) .
\end{aligned}
$$

Notice that $u \in A_{1}$ implies $u \in A_{q}$ for all $q>1$. Using Hölder's inequality and Lemma 4, we have

$$
\begin{aligned}
& K_{3}(y, r) \leq C u^{1-q}(B(y, r)){ }^{\left(1 / \alpha_{2}\right)-(1 / s)} \sum_{j=1}^{\infty} \frac{1}{\left|B\left(y, 2^{j+1} r\right)\right|} \int_{B\left(y, 2^{j+1} r\right)}|f(z)| \mathrm{d} z \times\|b\|_{\operatorname{Lip}_{\beta, u}} u(B(y, r))^{\beta / n} \frac{u(B(y, r))}{|B(y, r)|} \\
& \leq C\|b\|_{\operatorname{Lip}_{\beta, u}} u^{1-q}(B(y, r))^{\left(1 / \alpha_{2}\right)-(1 / s)} \sum_{j=1}^{\infty} \frac{1}{\left|B\left(y, 2^{j+1} r\right)\right|} \times\left(\int_{B\left(y, 2^{j+1} r\right)}|f(z)|^{p} u(z) \mathrm{d} z\right)^{1 / p}\left(\int_{B\left(y, 2^{j+1} r\right)} u(z)^{-p^{\prime} / p} d z\right)^{1 / p^{\prime}} \\
& \times u(B(y, r))^{\beta / n} \frac{u(B(y, r))}{|B(y, r)|} \\
& \leq C\|b\|_{\operatorname{Lip}_{\beta, u}} \sum_{j=1}^{\infty} u^{1-q}(B(y, r))^{\left(1 / \alpha_{2}\right)-(1 / s)}\left\|f \chi_{B\left(y, 2^{j+1} r\right)}\right\|_{L^{p}(u)} \times u\left(B\left(y, 2^{j+1} r\right)\right)^{-(1 / p)} u(B(y, r))^{\beta / n} \cdot \underset{x \in B}{\operatorname{essinf} u(x) .}
\end{aligned}
$$

From the fact that

$$
u(B(y, r))^{\beta / n} \leq u\left(B\left(y, 2^{j+1} r\right)\right)^{\beta / n},
$$

$$
\text { and }(1 / q)=(1 / p)-(\beta / n) \text {, we get }
$$

$$
\begin{aligned}
K_{3}(y, r) \leq & C\|b\|_{\operatorname{Lip}_{\beta, u}} \sum_{j=1}^{\infty} u^{1-q}(B(y, r))^{\left(1 / \alpha_{2}\right)-(1 / s)}\left\|f \chi_{B\left(y, 2^{j+1} r\right)}\right\|_{L^{p}(u)} \times u\left(B\left(y, 2^{j+1} r\right)\right)^{-(1 / q)} \underset{x \in B}{\operatorname{essinf}} u(x) \\
= & C\|b\|_{\operatorname{Lip}_{\beta, u}} \sum_{j=1}^{\infty} u^{1-q}\left(B\left(y, 2^{j+1} r\right)\right)^{\left(1 / \alpha_{1}\right)-(1 / p)-(1 / s)}\left\|f \chi_{B\left(y, 2^{j+1} r\right)}\right\|_{L^{p}(u)} \\
& \times \frac{u^{1-q}(B(y, r))^{\left(1 / \alpha_{2}\right)-(1 / q)-(1 / s)}}{u^{1-q}\left(B\left(y, 2^{j+1} r\right)\right)^{\left(1 / \alpha_{1}\right)-(1 / p)-(1 / s)}} u\left(B\left(y, 2^{j+1} r\right)\right)^{-(1 / q)} \times u^{1-q}(B(y, r))^{1 / q} \operatorname{essinf}_{x \in B} u(x) .
\end{aligned}
$$


Using the estimate

$$
\begin{aligned}
u^{1-q}(B(y, r))^{1 / q} \underset{x \in B}{\operatorname{essinf}} u(x) & =\left(\int_{B(y, r)} u^{1-q}(x) \mathrm{d} x\right)^{1 / q} \underset{x \in B}{\operatorname{essinf}} u(x) \\
& \leq\left(\underset{x \in B}{\operatorname{essinf}} u^{q}(x) \int_{B(y, r)} u^{1-q}(x) \mathrm{d} x\right)^{1 / q} \\
& \leq\left(\int_{B(y, r)} u(x) \mathrm{d} x\right)^{1 / q}=u(B(y, r))^{1 / q}
\end{aligned}
$$

we conclude that

$K_{3}(y, r) \leq C\|b\|_{\operatorname{Lip}_{\beta, u}} \sum_{j=1}^{\infty} u^{1-q}\left(B\left(y, 2^{j+1} r\right)\right)^{\left(1 / \alpha_{1}\right)-(1 / p)-(1 / s)}\left\|f \chi_{B\left(y, 2^{j+1} r\right)}\right\|_{L^{p}(u)} \times \frac{u^{1-q}(B(y, r))^{\left(1 / \alpha_{2}\right)-(1 / q)-(1 / s)}}{u^{1-q}\left(B\left(y, 2^{j+1} r\right)\right)^{\left(1 / \alpha_{1}\right)-(1 / p)-(1 / s)}}\left(\frac{u(B(y, r))}{u\left(B\left(y, 2^{j+1} r\right)\right)}\right)^{1 / q}$.

Since $u^{1-q}, u \in A_{\infty}$, there exists a constant $\delta>0$ such that

$$
\begin{aligned}
& \frac{u^{1-q}(B(y, r))^{\left(1 / \alpha_{2}\right)-(1 / q)-(1 / s)}}{u^{1-q}\left(B\left(y, 2^{j+1} r\right)\right)^{\left(1 / \alpha_{1}\right)-(1 / p)-(1 / s)}} \leq C \frac{|B(y, r)|^{\delta\left(\left(1 / \alpha_{2}\right)-(1 / q)-(1 / s)\right)}}{\left|B\left(y, 2^{j+1} r\right)\right|^{\delta\left(\left(1 / \alpha_{2}\right)-(1 / q)-(1 / s)\right)}} \\
& \leq C\left(\frac{1}{2^{(j+1) n}}\right)^{\delta\left(\left(1 / \alpha_{2}\right)-(1 / q)-(1 / s)\right)} \\
& \left(\frac{u(B(y, r))}{u\left(B\left(y, 2^{j+1} r\right)\right)}\right)^{1 / q} \leq C \frac{|B(y, r)|^{\delta / q}}{\left|B\left(y, 2^{j+1} r\right)\right|^{\delta / q}} \leq C\left(\frac{1}{2^{(j+1) n}}\right)^{\delta / q}
\end{aligned}
$$

Hence, we have

$$
K_{3}(y, r) \leq C\|b\|_{\operatorname{Lip}_{\beta, u}} \sum_{j=1}^{\infty} u^{1-q}\left(B\left(y, 2^{j+1} r\right)\right)^{\left(1 / \alpha_{1}\right)-(1 / p)-(1 / s)}\left\|f \chi_{B\left(y, 2^{j+1} r\right)}\right\|_{L^{p}(u)} \times\left(\frac{1}{2^{(j+1) n}}\right)^{\delta\left(\left(1 / \alpha_{2}\right)-(1 / s)\right)} .
$$

As for $K_{4}(y, r)$, we have

$$
\begin{aligned}
& K_{4}(y, r) \leq C u^{1-q}(B(y, r))^{\left(1 / \alpha_{2}\right)-(1 / s)} \sum_{j=1}^{\infty} \frac{1}{\left|B\left(y, 2^{j+1} r\right)\right|} \times \int_{B\left(y, 2^{j+1} r\right)}\left|b(z)-b_{2^{j+1} B}\right||f(z)| \mathrm{d} z \\
& +C u^{1-q}(B(y, r))^{\left(1 / \alpha_{2}\right)-(1 / s)} \sum_{j=1}^{\infty} \frac{1}{\left|B\left(y, 2^{j+1} r\right)\right|} \times \int_{B\left(y, 2^{j+1} r\right)}|f(z)| \mathrm{d} z \cdot\left|b_{B}-b_{2^{j+1} B}\right| \\
& =: K_{5}(y, r)+K_{6}(y, r) \text {. }
\end{aligned}
$$


Journal of Function Spaces

9

It follows from Hölder's inequality, the fact that $u \in A_{1}$, and Lemma 4, then we have

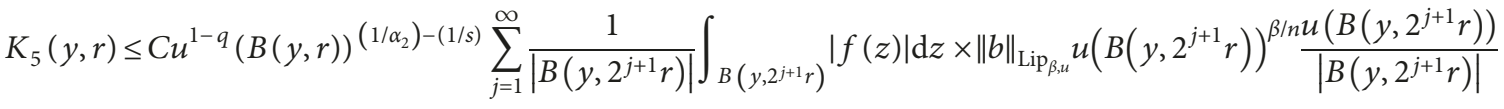

$$
\begin{aligned}
& \leq C\|b\|_{\operatorname{Lip}_{\beta, u}} \sum_{j=1}^{\infty} u^{1-q}(B(y, r)){ }^{\left(1 / \alpha_{2}\right)-(1 / s)}\left\|f \chi_{B\left(y, 2^{j+1} r\right)}\right\|_{L^{p}(u)} \times u\left(B\left(y, 2^{j+1} r\right)\right)^{-(1 / q)} \frac{u\left(B\left(y, 2^{j+1} r\right)\right)}{\left|B\left(y, 2^{j+1} r\right)\right|} \\
& \leq C\|b\|_{\operatorname{Lip}_{\beta, u}} \sum_{j=1}^{\infty} u^{1-q}\left(B\left(y, 2^{j+1} r\right)\right)^{\left(1 / \alpha_{1}\right)-(1 / p)-(1 / s)}\left\|f \chi_{B\left(y, 2^{j+1} r\right)}\right\|_{L^{p}(u)} \times \frac{u^{1-q}(B(y, r))^{\left(1 / \alpha_{1}\right)-(1 / p)-(1 / s)}}{u^{1-q}\left(B\left(y, 2^{j+1} r\right)\right)^{\left(1 / \alpha_{1}\right)-(1 / p)-(1 / s)}} \\
& \times u^{1-q}(B(y, r))^{1 / q} \cdot \underset{x \in 2^{j+1} B}{\operatorname{ess} \inf } u(x) u\left(B\left(y, 2^{j+1} r\right)\right)^{-(1 / q)} \\
& \leq C \mid\|b\|_{\operatorname{Lip}_{\beta, u}} \sum_{j=1}^{\infty} u^{1-q}\left(B\left(y, 2^{j+1} r\right)\right)^{\left(1 / \alpha_{1}\right)-(1 / p)-(1 / s)}\left\|f \chi_{B\left(y, 2^{j+1} r\right)}\right\|_{L^{p}(u)} \times \frac{u^{1-q}(B(y, r))\left(1 / \alpha_{2}\right)-(1 / q)-(1 / s)}{u^{1-q}\left(B\left(y, 2^{j+1} r\right)\right)^{\left(1 / \alpha_{1}\right)-(1 / p)-(1 / s)}}\left(\frac{u(B(y, r))}{u\left(B\left(y, 2^{j+1} r\right)\right.}\right)^{1 / q} \\
& \leq C\|b\|_{\operatorname{Lip}_{\beta, u}} \sum_{j=1}^{\infty} u^{1-q}\left(B\left(y, 2^{j+1} r\right)\right)^{\left(1 / \alpha_{1}\right)-(1 / p)-(1 / s)}\left\|f \chi_{B\left(y, 2^{j+1} r\right)}\right\|_{L^{p}(u)} \times\left(\frac{1}{2^{(j+1) n}}\right)^{\delta\left(\left(1 / \alpha_{2}\right)-(1 / s)\right)} .
\end{aligned}
$$

For the last term, applying Lemma 5 and the $A_{1}$ property, we have

$$
\begin{aligned}
& K_{6}(y, r) \leq C\|b\|_{\operatorname{Lip}_{\beta, u}} u^{1-q}(B(y, r))^{\left(1 / \alpha_{2}\right)-(1 / s)} \sum_{j=1}^{\infty}(j+1) u\left(B\left(y, 2^{j+1} r\right)\right)^{\beta / n} \times \underset{x \in B}{\operatorname{essinf}} u(x)\left\|f \chi_{B\left(y, 2^{j+1} r\right)}\right\|_{L^{p}(u)} u\left(B\left(y, 2^{j+1} r\right)\right)^{-(1 / p)} \\
& =C\|b\|_{\operatorname{Lip}_{\beta, u}} \sum_{j=1}^{\infty}(j+1) u^{1-q}\left(B\left(y, 2^{j+1} r\right)\right)^{\left(1 / \alpha_{1}\right)-(1 / p)-(1 / s)}\left\|f \chi_{B\left(y, 2^{j+1} r\right)}\right\|_{L^{p}(u)} \\
& \times \frac{u^{1-q}(B(y, r))^{\left(1 / \alpha_{2}\right)-(1 / q)-(1 / s)}}{u^{1-q}\left(B\left(y, 2^{j+1} r\right)\right)^{\left(1 / \alpha_{1}\right)-(1 / p)-(1 / s)}} u^{1-q}(B(y, r))^{1 / q} \times \underset{x \in B}{\operatorname{essinf}} u(x) \cdot u\left(B\left(y, 2^{j+1} r\right)\right)^{-(1 / q)} .
\end{aligned}
$$

By an argument similar to that used in estimating $K_{5}$, we get

$$
\begin{aligned}
K_{6}(y, r) \leq & C\|b\|_{\operatorname{Lip}_{\beta, u}} \sum_{j=1}^{\infty}(j+1) u^{1-q}\left(B\left(y, 2^{j+1} r\right)\right)^{\left(1 / \alpha_{1}\right)-(1 / p)-(1 / s)}\left\|f \chi_{B\left(y, 2^{j+1} r\right)}\right\|_{L^{p}(u)} \\
& \times \frac{u^{1-q}(B(y, r))\left(1 / \alpha_{2}\right)-(1 / q)-(1 / s)}{u^{1-q}\left(B\left(y, 2^{j+1} r\right)\right)^{\left(1 / \alpha_{1}\right)-(1 / p)-(1 / s)}}\left(\frac{u(B(y, r))}{u\left(B\left(y, 2^{j+1} r\right)\right)}\right)^{1 / q} \\
\leq & C\|b\|_{\operatorname{Lip}_{\beta, u}} \sum_{j=1}^{\infty}(j+1) u^{1-q}\left(B\left(y, 2^{j+1} r\right)\right)^{\left(1 / \alpha_{1}\right)-(1 / p)-(1 / s)}\left\|f \chi_{B\left(y, 2^{j+1} r\right)}\right\|_{L^{p}(u)} \times\left(\frac{1}{2^{(j+1) n}}\right)^{\delta\left(\left(1 / \alpha_{2}\right)-(1 / s)\right)} .
\end{aligned}
$$


Taking the $L^{s}(\nu)$-norm and using Minkowski's inequality, we get

$$
\begin{aligned}
& \left\|u^{1-q}(B(y, r))^{\left(1 / \alpha_{2}\right)-(1 / q)-(1 / s)}\right\|\left[b, T_{\Omega}\right] f \chi_{B(y, r)}\left\|_{L^{q}\left(u^{1-q}\right)}\right\|_{L^{s}(v)} \\
& \leq\left\|K_{1}\right\|_{L^{s}(v)}+\left\|K_{2}\right\|_{L^{s}(v)} \\
& \leq C\left\|u^{1-q}(B(y, 2 r))^{\left(1 / \alpha_{1}\right)-(1 / p)-(1 / s)}\right\| f \chi_{2 B}\left\|_{L^{p}(u)}\right\|_{L^{s}(v)} \\
& \quad+C \sum_{j=1}^{\infty}\left\|u^{1-q}\left(B\left(y, 2^{j+1} r\right)\right)^{\left(1 / \alpha_{1}\right)-(1 / p)-(1 / s)}\right\| f \chi_{B\left(y, 2^{j+1} r\right)}\left\|_{L^{p}(u)}\right\|_{L^{s}(v)} \\
& \quad \times(j+1)\left(\frac{1}{2^{(j+1) n}}\right)^{\delta\left(\left(1 / \alpha_{2}\right)-(1 / s)\right)} \\
& \leq C\|f\|_{\left(L^{p}, L^{s}\right)^{\alpha_{1}}\left(u, u^{1-q} ; v\right) .}
\end{aligned}
$$

Finally, by taking the supremum over all $r>0$, the proof is completed.

\section{Data Availability}

No data were used to support this study.

\section{Conflicts of Interest}

The authors declare that they have no conflicts of interest.

\section{Authors' Contributions}

All authors contributed equally to the writing of this paper. All authors read and approved the final manuscript.

\section{Acknowledgments}

The authors thank Prof. Xinfeng Wu for his very helpful comments. This work was supported in part by the National Natural Science Foundation of China (grant no. 11671397).

\section{References}

[1] R. R. Coifman, R. Rochberg, and G. Weiss, "Factorization theorems for hardy spaces in several variables," The Annals of Mathematics, vol. 103, no. 3, pp. 611-635, 1976.

[2] S. Janson, "Mean oscillation and commutators of singular integral operators," Arkiv för Matematik, vol. 16, no. 1-2, pp. 263-270, 1978.

[3] H. Wang, "Some estimates for $\theta$-type calder on-Zygmund operators and linear commutators on certain weighted amalgam spaces," 2017, https://arxiv.org/abs/1701.07508.

[4] B. Hu and J. Gu, "Necessary and sufficient conditions for boundedness of some commutators with weighted Lipschitz functions," Journal of Mathematical Analysis and Applications, vol. 340, no. 1, pp. 598-605, 2008.

[5] N. Wiener, "On the representation of functions by trigonometrical integrals," Mathematische Zeitschrift, vol. 24, no. 1, pp. 575-616, 1926.

[6] N. Wiener, "Tauberian theorems," The Annals of Mathematics, vol. 33, no. 1, pp. 1-100, 1932.
[7] F. Holland, "Harmonic analysis on amalgams of $L^{p}$ and , $l^{q}$," Journal of the London Mathematical Society, vol. s2-10, no. 3, pp. 295-305, 1975.

[8] J. Stewart, "Fourier transforms of unbounded measures," Canadian Journal of Mathematics, vol. 31, no. 6, pp. 12811292, 1979.

[9] J. J. F. Fournier and J. Stewart, "Amalgams of $L^{p}$ and $l^{q}$," Bulletin of the American Mathematical Society, vol. 13, no. 1, pp. 1-22, 1985.

[10] I. Fofana, "Etude d'une classe d'espaces de fonctions contenant les espaces de Lorentz," Arkiv för Matematik, vol. 1, no. 2, pp. 29-50, 1988.

[11] R. Devore and R. Sharpley, "Maximal functions measuring smoothness," Memoirs of the American Mathematical Society, vol. 47, no. 293, pp. 663-673, 1984.

[12] J. Garcia-Cuerva, "Weighted $H^{p}$ spaces," Demonstratio Mathematica, vol. 162, pp. 1-56, 1979. 


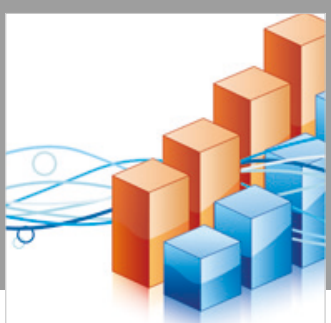

Advances in

Operations Research

\section{-n-m}
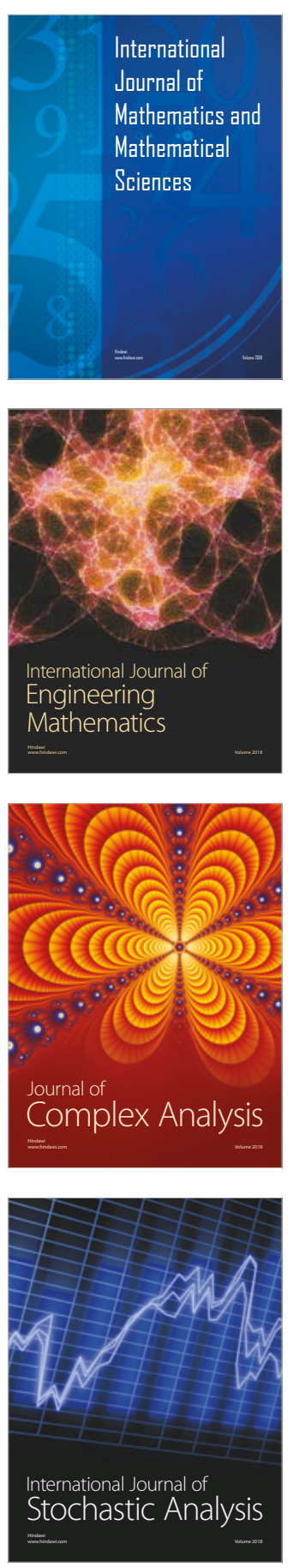
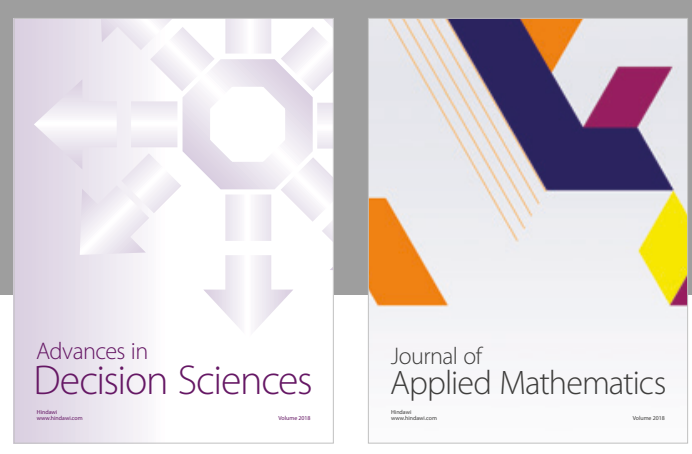

Journal of

Applied Mathematics
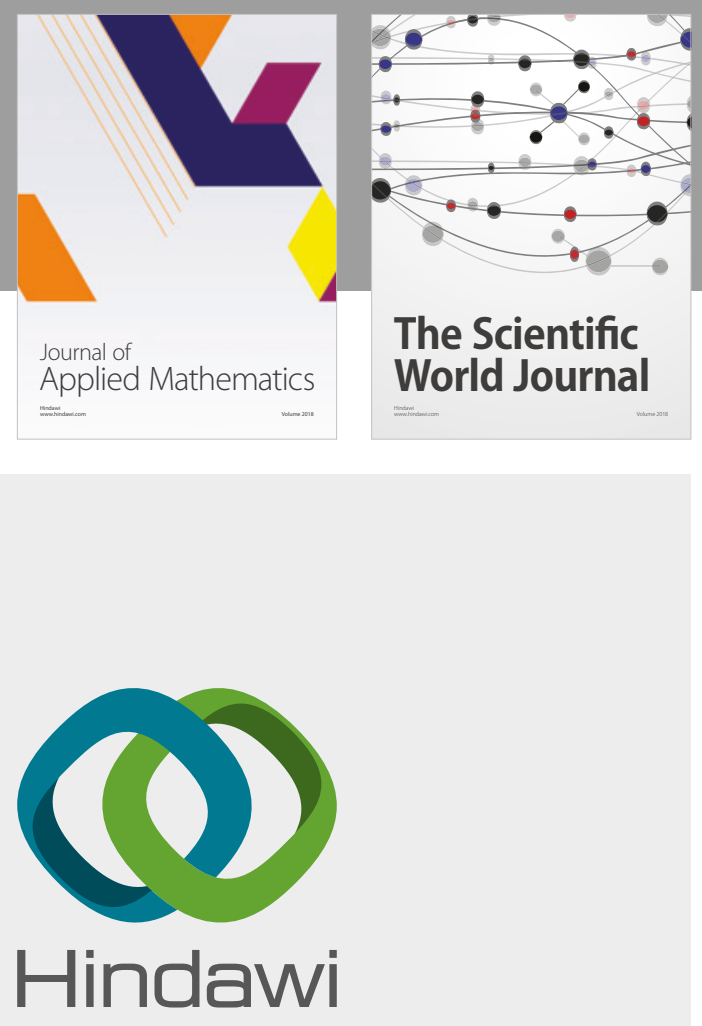

Submit your manuscripts at

www.hindawi.com

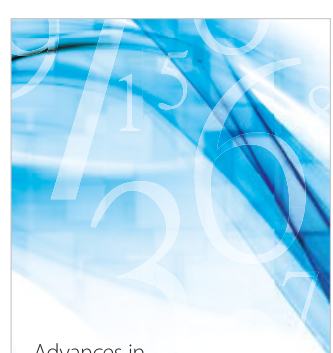

Advances in
Numerical Analysis
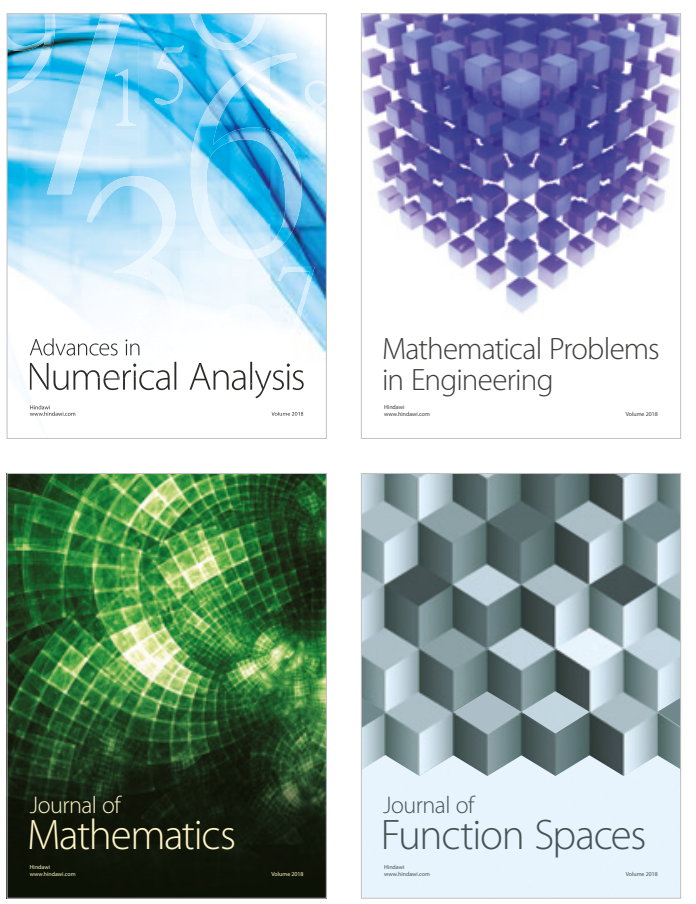

Mathematical Problems in Engineering

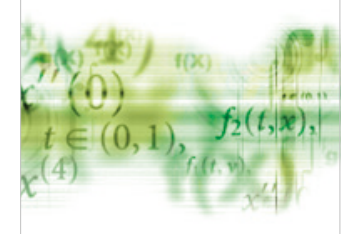

International Journal of

Differential Equations

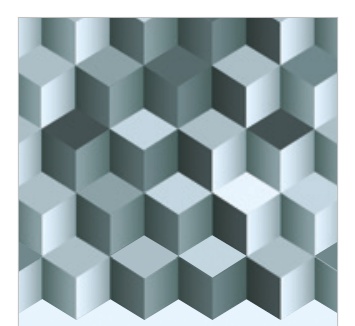

Journal of

Function Spaces

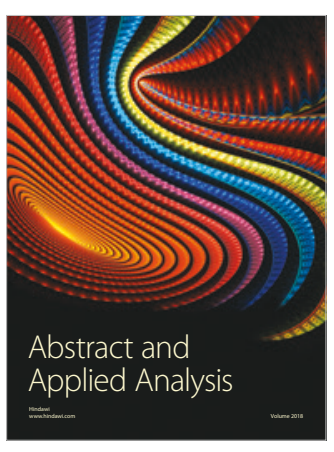

The Scientific

World Journal

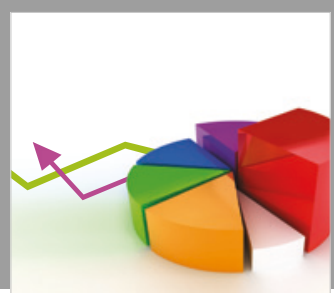

Journal of

Probability and Statistics
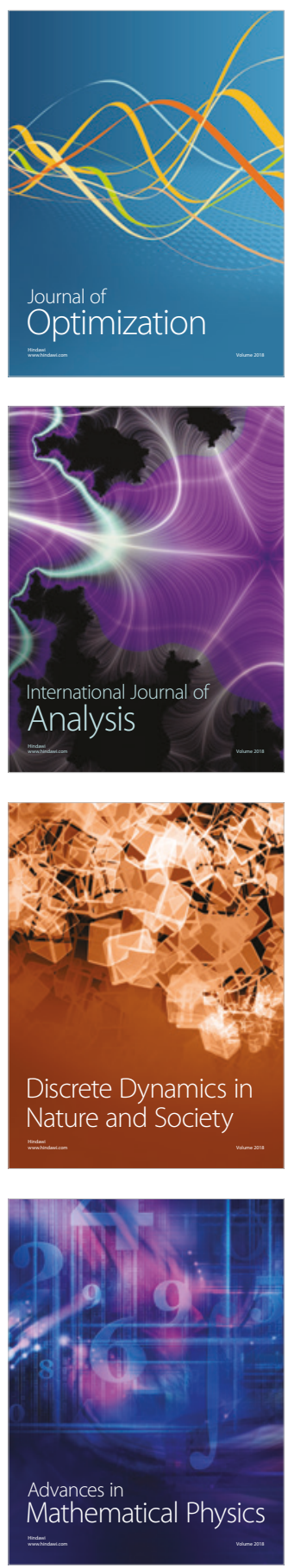